\title{
Restauración ecológica en plantaciones forestales de Eucalyptus globulus Labill y Acacia melanoxylon $R$. Br.
}

\section{Ecological restoration in forestry plantations of Eucalyptus globulus Labill and Acacia melanoxylon R. Br.}

\section{Restauração ecológica em plantações florestais de Eucalyptus globulus Labill e Acacia R. Br melanoxylon}

\author{
Lina Rocío Guio Cortés ${ }^{1}$, Jairo Solorza Bejarano² \& Leslie Yaneth Leal Mejía ${ }^{3}$ \\ 'Ingeniera Ambiental. 'Biólogo, Magister en Educación y Gestión Ambiental, Candidato a Magister en \\ Geografía de la Universidad Pedagógica y Tecnológica de Colombia -UPTC. ${ }^{3}$ Bióloga, Especialista en \\ Docencia Universitaria, Magister en Ciencias - Biología \\ ${ }_{1,3}$ Programa de Ingeniería Ambiental. Escuela de Ciencias Agrícolas, Pecuarias y del Medio \\ Ambiente - ECAPMA. Universidad Nacional Abierta y a Distancia -UNAD, Calle 14 sur No. 14-23 \\ piso 5. Bogotá, Colombia. ²Programa de Investigación en Restauración Ecológica. Subdirección \\ Científica. Jardín Botánico José Celestino Mutis. Bogotá. Colombia. \\ 1Irguioc1601@gmail.com, ${ }^{2}$ jsolorza@jbb.gov.co, ${ }^{3}$ leslie.leal@unad.edu.co
}

\begin{abstract}
Resumen
El aumento en la degradación de las condiciones naturales del ecosistema de los cerros orientales de Bogotá, Colombia, ha promovido la generación de nuevas estrategias que permitan restaurar ecosistemas de forma integral y adecuada, de acuerdo con sus características. Los escenarios de plantaciones forestales de Eucalyptus globulus Labill y Acacia melanoxylon $\mathrm{R}$. Br han sido intervenidos con procesos de restauración ecológica, generando una disminución en el impacto ocasionado al suelo por los fuertes procesos erosivos inherentes a estas especies. En este artículo de investigación se hace una revisión de los casos reportados de restauración ecológica realizados en $E$. globulus Labill y $A$. melanoxylon $R$. $B r$, con el fin de identificar actividades que puedan complementar la gestión realizada en restauración ecológica en Bogotá D.C. Se concluye que las actividades de restauración ecológica se deben desarrollar siguiendo
\end{abstract}

un orden lógico teniendo en cuenta el principal objetivo de esta estrategia. Resumiendo las actividades de restauración ecológica deben iniciar con una caracterización de las especies que se encuentran en la zona y la vegetación típica. Posteriormente se deben implementar las estrategias más adecuadas acorde con las características de la región, con el fin de reintroducir las especies nativas, de forma paulatina. Es necesario realizar un seguimiento y control constante con el fin de procurar el mayor éxito en las actividades de restauración realizadas.

Palabras clave: restauración ecológica, plantaciones exóticas, degradación, Eucalyptus globulus Labill, Acacia melanoxylon $\mathrm{R}$. Br.

\section{Abstract}

The rise in the deterioration of natural conditions associated with the ecosystem in the Eastern 
Mountains of Bogotá, has generated new strategies that allow restoring ecosystems in an integral and appropriate way, according to their characteristics. The scenarios in the forestry plantations of Eucalyptus globulus Labill and Acacia melanoxylon $\mathrm{R}$. $\mathrm{Br}$ are cultivated, They have been intervened with ecological restoration processes reducing the impact on the ground, due to the strong erosive processes inherent to these species. In this article, a summary of the reported cases of the ecological restoration made on E. globulus Labill and A. melanoxylon $\mathrm{R}$. $\mathrm{Br}$, is conduced in order to identify activities that complement the work done on the ecological restoration in Bogotá D. C. It was concluded that the activities of ecological restoration must be developed in a logical order taking into account the main objective of this strategy. The activities of ecological restoration must start with a characterization of the species located in the area and the typical vegetation. Subsequently the most appropriate strategies must be implemented in line with the characteristics of the region in order to reintroduce the native species, in a gradual way. It is necessary to perform a follow-up and constant monitoring in order to ensure the greatest success in restoration activities undertaken.

Key-words: ecological restoration, exotic plantations, degradation, Eucalyptus globulus Labill, Acacia melanoxylon $\mathrm{R}$. Br.

\section{Resumo}

O aumento na degradação das condições naturais do ecossistema das montanhas do leste de Bogotá, Colômbia, tem promovido a criação de novas estratégias para restaurar os ecossistemas forma abrangente e apropriada, de acordo com suas características. Cenários de plantações florestais de Eucalyptus globulus Labill e Acacia melanoxylon $\mathrm{R}$. Br foram operados com os processos de restauração ecológica, resultando em uma diminuição do impacto sobre o solo causada pelos fortes processos de erosão inerentes a estas espécies. Neste trabalho de pesquisa uma revisão dos casos relatados de restauração ecológica feita em E. globulus Labill e A. melanoxylon $\mathrm{R}$. $\mathrm{Br}$, a fim de identificar as actividades que podem complementar a gestão realizada em restauração ecológica em Bogotá DC é Conclui-se que as atividades de restauração ecológica deve ser desenvolvido em uma ordem lógica tendo em conta o objectivo principal desta estratégia. Resumindo as actividades de restauração ecológica deve começar com uma caracterização das espécies encontradas na área e vegetação típica. Em seguida, devem ser implementadas as estrategias mais adequadas de acordo com as características da região, a fim de reintroduzir espécies nativas, gradualmente. É necessário fazer um seguimento e monitoramento constante, a fim de garantir o sucesso em atividades de restauração realizadas.

Palavras-chave: restauração ecológica, as plantações de exóticas, de degradação, Eucalyptus globulus Labill, Acacia melanoxylon $\mathrm{R}$. Br.

\section{Introducción}

La degradación y alteración de la mayor parte de coberturas vegetales nativas de Colombia ha ocasionado diferentes impactos a nivel energético, ambiental, social y económico. (Personería de Bogotá, 2007). En los últimos años se ha evidenciado la degradación de diferentes ecosistemas a nivel mundial, ocasionada principalmente por factores como la transformación y perdida de ecosistemas, sobreexplotación de los recursos naturales, introducción de especies exóticas y contaminación ambiental. (Plan Nacional de Restauración, 2012).

A mediados del siglo XIX y principios del siglo XX, Bogotá fue testigo de la deforestación y degradación de los ecosistemas de los cerros orientales, ante el uso y explotación de los principales recursos naturales de la región (Osorio, 2009). Como respuesta a la búsqueda de materias primas que 
permitieran la satisfacción de las necesidades básicas de vida, la población bogotana extrajo leña, carbón vegetal y encenillo, en cantidades moderadas que con la acumulación del tiempo y el constante crecimiento demográfico, ocasionó la disminución de coberturas vegetales y por ende, la aparición de zonas desnudas en las montañas. (Alcaldía Mayor de Bogotá, 2006)

Igualmente, durante esta época la aparición de la alfarería y minería artesanal ocasionó impactos ambientales en las características del suelo y las condiciones naturales de los ecosistemas, debido a que estas actividades requerían la remoción de la cobertura vegetal de la zona a explotar, generando problemas de erosión y baja fertilidad de las zonas intervenidas. (Osorio, 2009). Ante esta situación, las instituciones gubernamentales establecieron mecanismos y estrategias tendientes a la recuperación y protección de las zonas naturales de la región, entre las cuales se enfatizó en la necesidad de reforestar los cerros orientales de la ciudad, para lo cual, se emplearon especies vegetales foráneas como Eucalyptus spp., A. spp. y Pinus spp., teniendo en cuenta su tasa de crecimiento y los beneficios económicos que se podría obtener de estas (Wiesner, 2007).

EI INDERENA generó el acuerdo número 30 de 1976 (INDERENA, 1976), como una de las estrategias orientadas a la recuperación de las condiciones naturales de los cerros orientales, mediante el cual se establece esta zona como área de reserva forestal y delega la responsabilidad de su protección y mantenimiento en la entonces denominada Corporación Autónoma Regional de la Sabana de Bogotá y de los Valles de Ubaté y Chiquinquirá. (Alcaldía Mayor de Bogotá, 2006). Sin embargo, y a pesar de la proclamación del acuerdo 30 como norma nacional mediante la Resolución 76 de 1977 del Ministerio de Agricultura, en los años posteriores se realizaron una serie de construcciones viales y de viviendas, que intervenían varias áreas pertenecientes a la reserva forestal, aumentando los impactos ambientales de la zona (Alcaldía Mayor de Bogotá, 2006).
Posterior a esto, durante los años ochenta, se generó un crecimiento significativo en la densidad poblacional ubicada en barrios ilegales construidos en los cerros orientales de Bogotá, como los barrios Juan XXIII, las Acacias y, sobre la vía la Calera, los barrios San Isidro, San Luis y la Esperanza (Instituto Distrital de Cultura y Turismo, 2004). Así mismo, durante la década de los 1990 a 2000, continuó la aparición de nuevos barrios, nuevos proyectos de vivienda y establecimiento de canteras (Wiesner, 2007). En el año 2005, mediante la resolución 0463 de 2005 (Ministerio de Ambiente, Vivienda y Desarrollo Territorial, 2005), se redefinieron los límites establecidos previamente como zonas de reserva forestal de los cerros orientales de Bogotá, dejando como consecuencia la reducción en 973 ha de la zona considerada como reserva forestal, las cuales fueron incluidas como zonas pertenecientes al área urbana de Bogotá.

Actualmente, los cerros orientales de Bogotá se han recuperado paulatinamente de la pérdida de la cobertura vegetal, aunque el crecimiento de especies foráneas ha ocasionado impactos ambientales, tales como erosión del suelo y alteración de las características naturales del ecosistema. (Instituto Distrital de Cultura y Turismo, 2004). Por esta razón, y ante el crecimiento de la preocupación por el equilibrio y conservación del ambiente, se han establecido nuevas estrategias tendientes a la recuperación y restauración de las condiciones naturales de los ecosistemas típicos de esta región (Wiesner, 2007).

La restauración ecológica es una de las principales estrategias de recuperación de ecosistemas, ya que emplea herramientas acordes a los requerimientos a nivel ambiental, social y económico de la zona afectada. (Plan Nacional de Restauración, 2012). En esta investigación, se analizó la información disponible sobre actividades de restauración ecológica que se han desarrollado en escenarios de plantaciones exóticas, que puedan adaptarse y utilizarse como acciones de restauración en los planes que se están 
desarrollando para la recuperación de ecosistemas en los cerros orientales de Bogotá D.C.

\section{Materiales y métodos}

En esta investigación se realizó una revisión bibliográfica a nivel nacional e internacional de diferentes fuentes de información de índole científica, tales como revistas indexadas y repositorios de entidades científicas reconocidas; empleando como tema central para la búsqueda escenarios de restauración ecológica en zonas de plantaciones exóticas, especialmente E. globulus Labill y A. melanoxylon $\mathrm{R}$. Br.

\section{Resultados y discusión}

Se encontraron diversos artículos y publicaciones de índole científica relacionados con actividades de restauración ecológica en escenarios de plantaciones forestales exóticas, permitiendo de esta manera realizar un acercamiento y recopilación sobre las metodologías, actividades y resultados obtenidos durante la implementación de dichas investigaciones. En la Tabla 1, se presentan los aspectos relevantes de 20 estudios de caso revisados, organizados por continente y teniendo en cuenta los países que presentan mayor número de estudios de caso en restauración ecológica:

Tabla 1. Aspectos relevantes de los estudios de caso de restauración ecológica considerados.

\begin{tabular}{|c|c|c|c|c|}
\hline Continente & País & Estudio de caso & Aspectos comunes & Aspectos diferentes \\
\hline \multirow{5}{*}{$\begin{array}{l}\text { América } \\
\text { del Sur }\end{array}$} & & $\begin{array}{l}\text { Efectos de las plantaciones } \\
\text { de E. globulus Labill en la } \\
\text { fuente hídrica del este de } \\
\text { la Cordillera de la Costa de } \\
\text { Biobío. }\end{array}$ & $\begin{array}{l}\text { Impactos ambientales ocasio- } \\
\text { nados por las plantaciones fo- } \\
\text { restales de E. globulus Labill. }\end{array}$ & $\begin{array}{l}\text { Presenta seguimiento } \\
\text { a las variables de caudal } \\
\text { y precipitación que } \\
\text { afecten la fuente hídrica } \\
\text { estudiada. }\end{array}$ \\
\hline & Chile & $\begin{array}{l}\text { Estudio de las propiedades } \\
\text { hidrológicas del suelo y } \\
\text { características de la expor- } \\
\text { tación de sedimentos en } \\
\text { una microcuenca, en dos } \\
\text { escenarios de plantaciones; } \\
\text { La primer zona de estudio } \\
\text { con plantaciones de bosque } \\
\text { nativo y plantaciones de E. } \\
\text { globulus Labill. }\end{array}$ & $\begin{array}{l}\text { Seguimiento puntual a los } \\
\text { efectos ocasionados por las } \\
\text { plantaciones exóticas en el } \\
\text { suelo y las variables hidro- } \\
\text { lógicas, teniendo en cuenta } \\
\text { la comparación con la zona } \\
\text { de estudio con plantaciones } \\
\text { nativas de la región. }\end{array}$ & $\begin{array}{l}\text { Comparación de las ca- } \\
\text { racterísticas de una zona } \\
\text { con plantaciones nativa } \\
\text { y otra con plantaciones } \\
\text { exóticas de E. globulus } \\
\text { Labill. }\end{array}$ \\
\hline & \multirow{3}{*}{ Colombia } & $\begin{array}{l}\text { Caracterización de las } \\
\text { zonas que presentan } \\
\text { plantaciones exóticas en el } \\
\text { Distrito Capital. }\end{array}$ & $\begin{array}{l}\text { Actividades necesarias para } \\
\text { realizar la restauración ecoló- } \\
\text { gica de zonas con plantacio- } \\
\text { nes exóticas. }\end{array}$ & $\begin{array}{l}\text { Presenta las actividades } \\
\text { y competencias de las } \\
\text { diferentes entidades dis- } \\
\text { tritales en la participación } \\
\text { de las actividades de } \\
\text { restauración ecológica. }\end{array}$ \\
\hline & & $\begin{array}{l}\text { Se evaluó la capacidad de } \\
\text { regeneración de Acacia } \\
\text { melanoxylon } \mathrm{R} \text {. Br, en sitios } \\
\text { donde se han implementa- } \\
\text { do actividades de restaura- } \\
\text { ción ecológica. }\end{array}$ & $\begin{array}{l}\text { Evaluación de los efectos } \\
\text { ocasionados al ambiente por } \\
\text { la regeneración de la especie } \\
\text { en estudio. }\end{array}$ & $\begin{array}{l}\text { Presentan el seguimiento } \\
\text { a las variables de creci- } \\
\text { miento de los individuos } \\
\text { empleados en las áreas } \\
\text { intervenidas. }\end{array}$ \\
\hline & & $\begin{array}{l}\text { Impactos ambientales } \\
\text { ocasionados por la intro- } \\
\text { ducción de individuos de A. } \\
\text { melanoxylon } \mathrm{R} \text {. Br, e zonas } \\
\text { ganaderas del Cauca. }\end{array}$ & $\begin{array}{l}\text { Efectos ocasionados por la } \\
\text { siembra de individuos de } \mathrm{A} \text {. } \\
\text { melanoxylon } \mathrm{R} \text {. Br y su rela- } \\
\text { ción con las demás especies } \\
\text { vegetales. }\end{array}$ & $\begin{array}{l}\text { Uso de la Acacia como } \\
\text { alternativa para la rege- } \\
\text { neración de ciertas } \\
\text { características del suelo. }\end{array}$ \\
\hline
\end{tabular}




\begin{tabular}{|c|c|c|c|c|}
\hline Continente & País & Estudio de caso & Aspectos comunes & Aspectos diferentes \\
\hline \multirow{6}{*}{$\begin{array}{l}\text { América } \\
\text { del Sur }\end{array}$} & \multirow{3}{*}{ Colombia } & $\begin{array}{l}\text { Presenta la planeación } \\
\text { e implementación de las } \\
\text { actividades de restauración } \\
\text { ecológica en zonas aleda- } \\
\text { ñas a los Cerros orientales } \\
\text { de la ciudad de Bogotá, } \\
\text { interviniendo principalmen- } \\
\text { te las especies E. globulus } \\
\text { Labill y A. melanoxylon R. } \\
\text { Br, }\end{array}$ & $\begin{array}{l}\text { Establece las actividades de } \\
\text { restauración ecológica y las } \\
\text { técnicas empleadas para la } \\
\text { dispersión de semillas en los } \\
\text { sitios de estudio. }\end{array}$ & $\begin{array}{l}\text { Inclusión de la pobla- } \\
\text { ción del lugar durante la } \\
\text { implementación de las } \\
\text { actividades de restaura- } \\
\text { ción ecológica. }\end{array}$ \\
\hline & & $\begin{array}{l}\text { Presenta las actividades } \\
\text { de restauración ecológica } \\
\text { que se implementaron en } \\
\text { el predio de la presa seca } \\
\text { cantarrana. }\end{array}$ & $\begin{array}{l}\text { Se controlaron especies como } \\
\text { E. globulus Labill y A. mela- } \\
\text { noxylon R. Br. }\end{array}$ & $\begin{array}{l}\text { No se evidencia ninguna } \\
\text { diferencia con las carac- } \\
\text { terísticas de los estudios } \\
\text { de casos nombrados } \\
\text { anteriormente. }\end{array}$ \\
\hline & & $\begin{array}{l}\text { Se estudia las consecuen- } \\
\text { cias positivas y negativas } \\
\text { de emplear la estrategia de } \\
\text { nucleación para la restaura- } \\
\text { ción ecológica en el parque } \\
\text { del Embalse del Neusa. }\end{array}$ & $\begin{array}{l}\text { Selección de las especies } \\
\text { vegetales acorde con las ca- } \\
\text { racterísticas de la vegetación } \\
\text { nativa de la región. }\end{array}$ & $\begin{array}{l}\text { Las especies vegeta- } \\
\text { les empleadas para } \\
\text { la restauración fueron } \\
\text { organizadas en núcleos, } \\
\text { y plantadas de forma es- } \\
\text { tratégica de tal forma que } \\
\text { se facilite la dispersión } \\
\text { de semillas }\end{array}$ \\
\hline & \multirow[t]{2}{*}{ Uruguay } & $\begin{array}{l}\text { Estima las tasas de erosión } \\
\text { del suelo ocasionados por } \\
\text { las plantaciones exóticas } \\
\text { de Eucalyptus globulus } \\
\text { Labill, empleando el modelo } \\
\text { USLE/RUSLE (Ecuación } \\
\text { Universal de Perdida de } \\
\text { Suelo/Ecuación Universal } \\
\text { de Perdida de Suelo Revi- } \\
\text { sada) }\end{array}$ & $\begin{array}{l}\text { Presenta los efectos de las } \\
\text { plantaciones exóticas sobre } \\
\text { las características naturales } \\
\text { del suelo. }\end{array}$ & $\begin{array}{l}\text { Cuantifica la degradación } \\
\text { del suelo a través de la } \\
\text { estimación de la erosión } \\
\text { generada por las planta- } \\
\text { ciones exóticas. }\end{array}$ \\
\hline & & $\begin{array}{l}\text { Impactos ocasionados por } \\
\text { las plantaciones de Eu- } \\
\text { calyptus spp., en diferentes } \\
\text { áreas de Uruguay. }\end{array}$ & $\begin{array}{l}\text { Se realizó seguimiento y con- } \\
\text { trol de las variables principales } \\
\text { del suelo como pH, acidez } \\
\text { intercambiable, humedad rela- } \\
\text { tiva, carbono orgánico, etc. }\end{array}$ & $\begin{array}{l}\text { Se caracterizan los } \\
\text { diferentes tipos de suelos } \\
\text { en los que se realiza el } \\
\text { estudio }\end{array}$ \\
\hline & Venezuela & $\begin{array}{l}\text { El estudio se encamino en } \\
\text { la comparación y determi- } \\
\text { nación de los efectos oca- } \\
\text { sionados a cuatro suelos } \\
\text { de ladera diferentes; los } \\
\text { bosques húmedos, sabanas } \\
\text { secundarias, suelos con } \\
\text { plantaciones de pinos y } \\
\text { Eucalyptus globulus Labill. }\end{array}$ & $\begin{array}{l}\text { Se determinan variables } \\
\text { importantes para conocer la } \\
\text { calidad del suelo y de la mate- } \\
\text { ria orgánica. }\end{array}$ & $\begin{array}{l}\text { Se determinaron las } \\
\text { características de los } \\
\text { microorganismos de } \\
\text { cada uno de los suelos } \\
\text { estudiados. }\end{array}$ \\
\hline
\end{tabular}




\begin{tabular}{|c|c|c|c|c|}
\hline Continente & País & Estudio de caso & Aspectos comunes & Aspectos diferentes \\
\hline \multirow{2}{*}{$\begin{array}{l}\text { América } \\
\text { del Norte }\end{array}$} & \multirow{2}{*}{ México } & $\begin{array}{l}\text { Aspectos ambientales } \\
\text { ocasionados por el estable- } \\
\text { cimiento de plantaciones de } \\
\text { Eucalyptus globulus Labill } \\
\text { en gran escala en áreas } \\
\text { tropicales de México. }\end{array}$ & $\begin{array}{l}\text { Afectaciones ocasionadas al } \\
\text { ambiente por las plantaciones } \\
\text { de Eucalyptus globulus Labill }\end{array}$ & $\begin{array}{l}\text { Presenta medidas de } \\
\text { mitigación de las afec- } \\
\text { taciones del ambiente } \\
\text { por las plantaciones } \\
\text { de Eucalyptus globulus } \\
\text { Labill, aplicables a las } \\
\text { características de los } \\
\text { ecosistemas tropicales } \\
\text { de México }\end{array}$ \\
\hline & & $\begin{array}{l}\text { Aspectos ambientales } \\
\text { ocasionados por el estable- } \\
\text { cimiento de plantaciones de } \\
\text { Eucalyptus globulus Labill } \\
\text { en gran escala en áreas } \\
\text { tropicales de México. }\end{array}$ & $\begin{array}{l}\text { Afectaciones ocasionadas al } \\
\text { ambiente por las plantaciones } \\
\text { de Eucalyptus globulus Labill }\end{array}$ & $\begin{array}{l}\text { Presenta medidas de } \\
\text { mitigación de las afec- } \\
\text { taciones del ambiente } \\
\text { por las plantaciones } \\
\text { de Eucalyptus globulus } \\
\text { Labill, aplicables a las } \\
\text { características de los } \\
\text { ecosistemas tropicales } \\
\text { de México }\end{array}$ \\
\hline \multirow{6}{*}{ Europa } & \multirow{6}{*}{ España } & $\begin{array}{l}\text { Estado de las zonas de Es- } \\
\text { paña que tienen plantacio- } \\
\text { nes exóticas y actividades } \\
\text { para recuperar la vegeta- } \\
\text { ción nativa. }\end{array}$ & $\begin{array}{l}\text { Efectos e impactos ocasio- } \\
\text { nados por la presencia de } \\
\text { plantaciones exóticas en un } \\
\text { área determinada. }\end{array}$ & $\begin{array}{l}\text { Es un diagnostico gene- } \\
\text { ral de la situación de las } \\
\text { plantaciones exóticas, las } \\
\text { actividades propuestas } \\
\text { no han sido implemen- } \\
\text { tadas }\end{array}$ \\
\hline & & $\begin{array}{l}\text { Implicaciones de las } \\
\text { plantaciones de Eucalyptus } \\
\text { globulus Labill empleadas } \\
\text { para la reforestación, en el } \\
\text { suelo. }\end{array}$ & $\begin{array}{l}\text { Efectos ocasionados por las } \\
\text { plantaciones en el Eucalyptus } \\
\text { globulus Labill., modificacio- } \\
\text { nes en el pH, conductividad, } \\
\text { humedad, etc. }\end{array}$ & $\begin{array}{l}\text { Realiza seguimiento a } \\
\text { través de los años de las } \\
\text { zonas estudiadas. }\end{array}$ \\
\hline & & $\begin{array}{l}\text { Efectos alelopáticos oca- } \\
\text { sionados por Eucalyptus } \\
\text { globulus Labill, sobre las } \\
\text { plantaciones nativas de la } \\
\text { región Santiago de Com- } \\
\text { postela. }\end{array}$ & $\begin{array}{l}\text { Impactos ambientales genera- } \\
\text { dos por la alelopatía ocasiona- } \\
\text { da por la especie Eucalyptus } \\
\text { globulus Labill, }\end{array}$ & $\begin{array}{l}\text { Presenta también los } \\
\text { efectos ocasionados en } \\
\text { la interacción con demás } \\
\text { comunidades vegetales }\end{array}$ \\
\hline & & $\begin{array}{l}\text { Afectaciones que se } \\
\text { ocasionan por la descom- } \\
\text { posición de la hojarasca de } \\
\text { Eucalyptus globulus Labill } \\
\text { en dos zonas con carac- } \\
\text { terísticas diferentes; un } \\
\text { bosque ripario en nutrientes } \\
\text { y uno pobre, realizando la } \\
\text { investigación también con } \\
\text { la introducción de vegeta- } \\
\text { ción nativa. }\end{array}$ & $\begin{array}{l}\text { Cambios en las características } \\
\text { de los suelos de cada uno de } \\
\text { los escenarios estudiados, } \\
\text { y los efectos ocasionados } \\
\text { en cada una de las especies } \\
\text { vegetales empleadas. }\end{array}$ & $\begin{array}{l}\text { Se realiza el estudio de } \\
\text { caso únicamente con los } \\
\text { dos escenarios estudia- } \\
\text { dos. }\end{array}$ \\
\hline & & $\begin{array}{l}\text { Presenta las actividades } \\
\text { de restauración ecológica } \\
\text { realizadas para la zona } \\
\text { desertificada de Albatera. }\end{array}$ & $\begin{array}{l}\text { Se implementaron actividades } \\
\text { características de restauración } \\
\text { ecológica en la zona afectada }\end{array}$ & $\begin{array}{l}\text { Se implementaron acti- } \\
\text { vidades enfocadas a la } \\
\text { prevención de la erosión } \\
\text { y desertificación de los } \\
\text { suelos. }\end{array}$ \\
\hline & & $\begin{array}{l}\text { Presenta un estado del } \\
\text { arte a nivel nacional sobre } \\
\text { los efectos sobre el suelo } \\
\text { que son atribuidos a las } \\
\text { plantaciones de Eucalyptus } \\
\text { globulus Labill, en España. }\end{array}$ & $\begin{array}{l}\text { Presenta los impactos am- } \\
\text { bientales positivos que se han } \\
\text { logrado determinar acorde } \\
\text { con los estudios técnicos y } \\
\text { científicos realizadas en áreas } \\
\text { de condiciones similares a los } \\
\text { sitios de estudio. }\end{array}$ & $\begin{array}{l}\text { Se basa en las percep- } \\
\text { ciones de la población de } \\
\text { las zonas de estudio, y } \\
\text { también se apoya de los } \\
\text { estudios técnicos que han } \\
\text { sido desarrollados con } \\
\text { anterioridad. }\end{array}$ \\
\hline
\end{tabular}


Las actividades de restauración ecológica cumplen un papel importante en la recuperación de las condiciones naturales tanto ambientales como ecológicas de los diferentes ecosistemas mundiales, a través del uso y mejoramiento continuo de técnicas y tecnologías diseñadas para recuperar de forma adecuada las condiciones naturales. (Capdevila, Iglesias, Orueta \& Zilleti, 2006). La presencia de plantaciones exóticas puede generar diversos cambios e impactos en las características naturales de un ecosistema determinado, generando cambios significativos en la vegetación, dinámica y características de los suelos, alteraciones en el régimen hídrico y modificaciones en el balance másico y energético de la biomasa de la región. (Vargas, León \& Díaz, 2009). Por esta razón, es importante verificar los cambios que pueden generarse en cada uno de los aspectos anteriormente mencionados.

\section{Efectos ocasionados por plantaciones exóti-} cas de E. globulus Labill y A. melanoxylon R. $\mathrm{Br}$, en el suelo

Acorde con investigaciones realizadas en Uruguay se ha determinado que las zonas en las que se realizan plantaciones de E. globulus Labill, con el fin de emplearlos como materia prima para la producción de papel, tienden a sufrir procesos de compactación debido al uso de maquinarias y la extracción de grandes cantidades de madera en camiones pesados, degradando paulatinamente sus características propias. (Ceccon \& Martínez, 1999). Así mismo, para algunas de estas plantaciones se realizan quemas controladas con el objetivo de retirar las especies de hierbas indeseables, limpiar el terreno y facilitar la explotación del suelo, quemando en muchas ocasiones los residuos de E. globulus Labill presentes en la zona, y generando a su vez, un aumento en la temperatura del suelo y generando un impacto en la impermeabilidad causando efectos negativos en el drenaje del suelo y disminuyendo la cantidad y calidad de los nutrientes presentes en él (Ceccon \& Martínez, 1999).
En Colombia se ha logrado detectar que la especie E. globulus Labill genera efectos adversos en la estabilidad del suelo en términos de estructura, realizando la comparación en zonas con dos climas diferentes; el primer estudio se realizó en condiciones de clima húmedo y el segundo en clima seco (Núñez, 2013). En la primera zona estudiada se logró evidenciar que las afectaciones ocurrían en un menor grado con respecto a la segunda, en la cual, además de la estabilidad se vieron afectadas otras características del suelo como la humedad relativa, densidad aparente y permeabilidad. (Rubiano \& Garzón, 1989). Las plantaciones forestales exóticas se caracterizan por ser cultivos que tienen altos requerimientos nutricionales, debido a las altas tasa de crecimiento de las mismas (Lobos, 2013). La baja capacidad de desarrollar procesos de reciclaje de nutrientes en los suelos aumenta sus requerimientos, lo que genera una baja fertilidad de los terrenos en los cuales se encuentran plantados (Osorio, 2009). Así mismo, E. globulus Labill por alelopatía puede ocasionar aumento en la acidez del suelo, disminuyendo la capacidad de absorción de los nutrientes del suelo por parte del resto de plantas (Wiesner, 2007). Investigaciones de la Universidad Nacional de Colombia, indican que las plantaciones de A. melanoxylon $\mathrm{R}$. Br cuentan con un amplio potencial de fijación de nitrógeno, mejorando de forma notoria las características y condiciones del suelo, aportando así mismo, amplias cantidades de materia orgánica. (Santiago \& Piedrahita, 2012).

\section{Efectos ocasionados por plantaciones exóti-} cas de E. globulus Labill y A. melanoxylon R. $\mathrm{Br}$, en el recurso hídrico

A nivel hídrico, las plantaciones de E. globulus Labill se caracterizan por las grandes cantidades de agua que requieren para su rápido crecimiento, aunque la cantidad de agua absorbida puede variar acorde según el tipo de raíz que presente el árbol (Ceccon \& Martínez, 1999). Igualmente, por las condiciones morfológicas de esta especie se disminuye la cantidad de agua lluvia que llega al suelo, minimizando de esta forma la cantidad de agua disponible para absorción; sin embargo, una 
de las principales características de los E. globulus Labill, es la capacidad de retener parte del agua que se puede presentar en la neblina, equilibrando la cantidad de agua necesaria para su crecimiento. (Huber, Iroume, Mohr \& Frene, 2010). Igualmente, las plantaciones de A. melanoxylon $\mathrm{R}$. Br, por sus características morfológicas pueden ocasionar un mayor consumo de agua, al requerir mayores cantidades para su adecuado desarrollo, dependiendo igualmente del tamaño de la estructura de los individuos. (Quiroz, Pincheira, Hernández, González, García \& Soto, 2014).

Efectos ocasionados por plantaciones exóticas de E. globulus Labill y A. melanoxylon R. $\mathrm{Br}$, en los factores bióticos de un ecosistema

En el ámbito biótico, uno de los principales efectos ocasionados por las plantaciones de E. globulus Labill es el deterioro de los hábitats de diferentes especies de animales pequeños, los cuales a su vez generan un desequilibrio en la cadena trófica propia del ecosistema. (Ballester, Arias, Cobián, López \& Vieitez, 1982). La introducción de especies exóticas puede modificar la calidad del agua, alterando las poblaciones de invertebrados acuáticos y la presión de depredación debida a las especies introducidas, variando los patrones de reproducción por la presencia de plantaciones (Plan Nacional de Restauración, 2012). Así mismo, los invertebrados se ven afectados por la presencia de plantaciones de E. globulus Labill deteniendo varios de sus procesos vitales para el equilibrio del ecosistema. (Ceccon \& Martínez, 1999). En Brasil, se logró determinar que la formación de humus en el suelo se detuvo por la baja presencia de oligoquetas en el municipio de Paraná, ocasionada por las excesivas plantaciones de E. globulus Labill en la zona. (Dionisio, 1994).

En Brasil, en la región de Espíritu Santo, se ha logrado determinar que a pesar de la existencia de una amplia zona de plantaciones exóticas de $E$. globulus Labill, se encontró gran variedad de especies animales a causa del adecuado manejo de la zona. (Pinilla \& Suarez, 1998). Así mismo, también es importante resaltar que el plantar especies exóticas en un área determinada, puede ocasionar cambios en el ecosistema nativo al introducir especies distintas y que pueden generar presión en el equilibrio del ecosistema. (Azula, 2012).

\section{Efectos ocasionados por plantaciones exóti- cas de E. globulus Labill y A. melanoxylon R. $\mathrm{Br}$, en la vegetación}

Teniendo en cuenta las afectaciones mencionadas anteriormente, se puede decir que las plantaciones de E. globulus Labill ejercen una amplia presión con relación a las especies vegetales que se encuentren aledañas, debido a que se genera una competencia por los recursos hídricos y nutrientes presentes en el suelo, dependiendo de los regímenes de lluvia de la región. (Delgado, Alliaume, García \& Hernández, 2006). Así mismo, se ha logrado evidenciar que el potencial alelopático del E. globulus Labill es mayor en sitios donde existen regímenes bajos de lluvia o donde se presenta de forma errática, ocasionando que las sustancias toxicas generadas por las plantaciones de E. globulus Labill no se diluyan. (Fierro, González, GaIlardo, Castro, 2009). Las plantaciones de E. globulus Labill generan mayores efectos alelopáticos sobre las plantaciones que son caracterizadas como gramíneas y herbáceas, teniendo en cuenta igualmente las condiciones climáticas de la región en estudio, ya que de contar con regímenes altos de lluvia se facilitaría la escorrentía superficial de las sustancias toxicas, de tal forma que permite el crecimiento de vegetación nativa en medio de las plantaciones de E. globulus Labill (Pinilla \& Suarez, 1998).

Además, el deterioro de las condiciones naturales de los territorios, especialmente de la densidad de plantación, luminosidad baja y poca cantidad de agua presente en el suelo, ha generado una disminución en el espacio disponible para la germinación y crecimiento de especies representativas de las plantaciones nativas. (Wiesner, 2007). En cuanto a las plantaciones de A. melanoxylon $\mathrm{R}$. Br, se logró determinar el potencial alelopático ocasionado a través de la recolección y dilución de la hojarasca producida por ésta, encontrando 
que presenta un gran potencial inhibidor de crecimiento, pero con el tiempo, este efecto disminuye notoriamente. (Souto, González \& Reigosa, 1993). Por otra parte, en estudios realizados en Galicia, se estableció que la especie A. melanoxylon $\mathrm{R}$. $\mathrm{Br}$ genera un potencial fuerte de inhibición de la germinación de las especies nativas de esta región. (Hussain, Gonzalez \& Reigosa, 2007). Por otra parte, las plantaciones de A. melanoxylon R. $\mathrm{Br}$, son empleadas como barreras vivas para el control de determinada vegetación, especialmente cuando se requiere la implementación de sitios silvopastoriles. (Arboleda, Tombe, Morales \& Vivas, 2012).

\section{Principales actividades de restauracion eco-} logica en escenarios de plantaciones exoticas de E. globulus Labill y A. melanoxylon R. Br, implementadas a nivel nacional e internacional Para implementar actividades de restauración ecológica de forma adecuada es necesario realizar una revisión y verificación de las características principales de la zona a restaurar, especialmente, para conocer las características, composición y funcionamiento del ecosistema que se degradó, los impactos que se ocasionaron y la magnitud del daño ocasionado; y por último es importante conocer las características de las especies nativas de la región. (Garcés, Peña, Cano-Santana, Villeda \& Orozco, 2009). Con el fin de establecer los aspectos físicos, ecológicos y sociales de las áreas a intervenir, se desarrollan encuestas y actividades tendientes a la recolección de esta información básica para el planteamiento de las estrategias de restauración ecológica, incluyendo la generación de planos y mapas cartográficos de la zona en estudio. (Carvallo, 2009). Posterior a esto, se establece la metodología que permita determinar el potencial de restauración de la zona estudiada, empleando técnicas, conceptos o estrategias, teniendo en cuenta la disponibilidad de nutrientes, energía, características climatológicas, geomorfológicas y geológicas del área. (Salamanca \& Camargo, 2000). Así mismo, cuando se requiera por la extensión de terreno a estudiar, se pueden dividir las áreas en zonas más pequeñas y plantear estrategias específicas para cada una acorde con la información recolectada de sus componentes físicos, bióticos y sociales. (Chirino, Vilagrosa, Bautista \& Vallejo, 2011).

Para continuar, es necesario establecer y definir el tipo de vegetación de la zona y sus respectivas características; cómo se relacionan entre sí, cuál es el estado de la cobertura y si existen o no factores que puedan generar alguna tensión sobre las coberturas vegetales. (Oyarzun, Frene, Lacrampe, Huber \& Herve, 2011). Así mismo, es importante identificar los diferentes usos de suelo de la zona, permitiendo de esta forma realizar un cruce entre las diferentes cartografías identificando las áreas donde se debe iniciar con las actividades de restauración ecológica. (Manrique, 2009). A partir de esta información se pueden definir las especies que se emplearán para la restauración ecológica, así como las técnicas y cantidades en que se emplearán. (Chirino, Vilagrosa, Bautista \& Vallejo, 2011). Como actividad principal para el establecimiento de las actividades de restauración ecológica, se deben realizar diferentes muestreos de las coberturas vegetales actuales de la zona de estudio, con el fin de conocer e identificar las plantaciones dominantes y los efectos que pudieran ocasionar en el ecosistema de estudio; analizando de igual forma, la fisionomía de estas plantaciones y clasificando el potencial de restauración en alto, medio o bajo acorde con sus características. (Manrique, 2009). Igualmente, se pueden emplear técnicas y actividades que permitan potenciar la presencia de las especies nativas de la región. (Chirino, Vilagrosa, Bautista \& Vallejo, 2011).

Para que las actividades de restauración ecológica sean eficientes se deben realizar limpiezas de las zonas a intervenir, en las cuales se retiren las plantaciones forestales exóticas que han ocasionado la alteración del equilibrio del ecosistema, y por ende, la degradación del mismo. (Domingo, 2010). También es importante que durante la limpieza y preparación del área, se recojan residuos orgánicos e inorgánicos que puedan afectar el desarrollo de las actividades. (Garcés, Peña, Cano- 
Santana, Villeda \& Orozco, 2009). Una de las alternativas más empleadas para la introducción de especies con fines de restauración ecológica, es la nucleación. Esta técnica consiste en organizar las especies a introducir en grupos estratégicos que permitan la aceleración de los procesos de dispersión incrementando el flujo ecológico entre las zonas que han sido intervenidas y las no intervenidas. (Clerici \& García, 2001). Así mismo, esta estrategia es recomendable para implementar en zonas extensas ya que disminuye los costos de implementación. (Contreras Rodríguez, Moreno Cárdenas \& Barrera Cataño, 2011).

Para la implementación de las estrategias de restauración es necesario que se tengan en cuenta todos los diferentes componentes de un ecosistema. Sin embargo, los principales aspectos a abordar son el suelo, la vegetación y el factor biótico del ecosistema; siendo cada uno de estos factores claves para el éxito de las estrategias de restauración ecológica a implementar. (Manrique, 2009). En el componente de suelo, es importante que se adecuen sus condiciones de tal forma que facilite la introducción de las estrategias a emplear. Por ejemplo, en las zonas en las que se presentan claros es importante establecer coberturas vegetales, coberturas muertas, procesos de fertilización con componentes orgánicos o procesos de encalados o quemas fertilizantes. Por otra parte, al existir suelos con procesos de erosión se pueden emplear coberturas vegetales, instalación de barreras vivas, creación de zanjas, emparejar el terreno y suavizar los taludes. (Camacho, Mejia, 2011). Así mismo, es importante que se controlen las condiciones de la zona al momento de introducir las especies verificando que las técnicas, tecnologías y herramientas empleadas al momento de realizar la siembra de las nuevas especies sean las adecuadas; para esto, es importante también que el suelo haya sido preparado con anterioridad a la siembra. (Chirino, Vilagrosa, Bautista \& Vallejo, 2011).

Por último, se debe plantear un programa de seguimiento a los individuos introducidos en la zona de estudio, con el fin de verificar las condiciones de su crecimiento y los efectos que se pudieron ocasionar en el comportamiento de la vegetación de la zona. (Chirino, Vilagrosa, Bautista \& Vallejo, 2011). A su vez, con la generación de este programa se puede verificar la dispersión y crecimiento de plantaciones exóticas que hayan podido quedar como residuos de las primeras fases de las actividades de restauración ecológica. (Garcés, Peña, Cano-Santana, Villeda \& Orozco, 2009).

\section{Conclusiones}

Las plantaciones forestales de E. globulus Labill y A. melanoxylon $R$. Br, han ocasionado procesos erosivos en los suelos de los cerros orientales de Bogotá, como consecuencia de sus características físicas y químicas. Las actividades de restauración ecológica que se desarrollan en los cerros orientales de Bogotá han tenido en cuenta la información sobre las actividades que se han desarrollado en otros ecosistemas para el control de estas especies. Aunque en la Vereda Venado de Oro se ha evidenciado la presencia de E. globulus Labill y A. melanoxylon R. Br, los procesos erosivos observados no presentan estados tan críticos como los expuestos en algunos de los documentos consultados para el desarrollo de este trabajo. Este hecho puede deberse en gran parte a la presencia de especies como Weinmannia spp, Clusia spp, Myrsine spp y Oreopanax spp; que por ser especies nativas de los cerros orientales, han amortiguado gran parte de los efectos negativos de las plantaciones de E. globulus Labill y A. melanoxylon $\mathrm{R}$. Br.

Las actividades de restauración ecológica se deben desarrollar siguiendo un orden lógico teniendo en cuenta el principal objetivo de esta estrategia. A manera de resumen se puede decir que las actividades de restauración ecológica deben iniciar con una caracterización de las especies que se encuentran en la zona y la vegetación que es típica de esta región. Posteriormente se deben implementar las estrategias más adecuadas acorde 
con las características de la región, con el fin de reintroducir las especies nativas, de forma paulatina. (Solorza, 2011).

La definición de las especies a introducir en cualquier zona afectada por la presencia de plantaciones foráneas o exóticas, debe realizarse teniendo en cuenta las características naturales del área intervenida, y las capacidades y características que pueden facilitar su dispersión y crecimiento, tales como composición del suelo, humedad, clima, etc. Por otra parte, siempre se debe procurar la preparación del terreno antes de la plantación de las especies nativas. (Rios, 2010).

Para terminar, después de realizar la siembra de las plantaciones nativas, es necesario realizar un seguimiento y control constante con el fin de procurar el mayor éxito en las actividades de restauración realizadas. Esta estrategia permite conocer las características de propagación de las especies y evaluar si se está realizando de forma adecuada y eficiente, de tal forma que se puedan realizar los correctivos necesarios.

\section{Literatura citada}

1. Alcaldía Mayor de Bogotá. (2006). Plan de Manejo Ambiental Audiencia CAR. Recuperado de: http://www.institutodeestudiosurbanos.info/dmdocuments/cendocieu/ coleccion_digital/Valorizacion_Parques_Naturales/ PMA_Cerros_Orientales-Alcaldia_Mayor-2006.pdf

2. Alcaldía Mayor de Bogotá. (2006). Cerros orientales de Bogota, territorio sostenible y patrimonio ambiental: Para sus pobladores actuales, el distrito, la región y la nación. Recuperado de: https://mesacerros.files.wordpress.com/2007/10/documento_para_el_alcaldez.pdf

3. Arboleda, D., Tombe, A., Morales-Velasco, S. \& VivasQuilla, J. (2012). Propuesta para el establecimiento de especies arbóreas y arbustivas con potencial forrajero en sistemas de producción ganadera del Trópico Alto Colombiano. Bio Agro.. 11(1), 154-163.

4. Azula, M. (2012). Caracterización del compost producido a partir de la biomasa de siete especies exóticas e invasoras seleccionadas en el proyecto de restauración ecológica. Recuperado de: :http://repositorio.jbb.gov. co/bitstream/001/500/4/Anexo\%203.pdf

5. Ballester, A., Arias, A., Cobián, B. López, E. \& Vieitez, E. (1982). Estudio de potenciales alelopáticos originados por Eucalyptus globulus Labill, Pinus pinaster Ait y Pinus radiata D. Pastos. 12 (2), $239-254$.
6. Camacho, C., Mejía, A. (2011). Restauración ecológica del campus de la Institución Universitaria Politécnico Grancolombiano. Poliantea. 7(12).243-270.

7. Capdevila, L., Iglesias, A., Orueta, J. \& Zilleti, B. (2006) Especies exóticas invasoras: Diagnostico y bases para la prevención y el manejo. Madrid: Sociedad Anónima de Fotocomposición.

8. Carvallo, G. (2009). Especies exóticas e invasiones biológicas. Ciencia Ahora. 23(12). 15-21.

9. Ceccon, E. \& Martínez, M. (1999). Aspectos ambientales referentes al establecimiento de plantaciones de eucalipto de gran escala en áreas tropicales: aplicación al caso de México. Revista de ciencia y tecnología de América Interciencia, 24(6), 352-359.

10. Chirino, E., Vilagrosa, A., Bautista, A. \& Vallejo, V. (2011). Un caso aplicado de restauración ecológica para combatir la desertificación en ecosistemas semiáridos degradados: El proyecto piloto de Albatera (España). Red Iberoamericana y del Caribe de Restauración Ecológica. 5 (1). 3-4.

11. Clerici, C. \& García, P. (2001). Aplicaciones del modelo USLE/RUSLE para estimar perdidas de suelo por erosión en Uruguay y la región sur de la cuenca del rio de la plata. Agrociencia. (1) 902-103.

12. Contreras Rodríguez, S., Moreno Cárdenas, A. \& Barrera Castaño, J. (2011). La nucleación como una estrategia de restauración ecológica en el parque forestal del embalse del Neusa (Cundinamarca - Colombia). Red Iberoamericana y del Caribe de Restauración Ecologica. 5 (1). 5-6.

13. Delgado, S., Alliaume, F., García, P. \& Hernández, J. (2006). Efecto de las plantaciones de Eucalyptus sp. sobre el recurso suelo en Uruguay. Agrociencia. 10(2), 95 - 107.

14. Dionisio, J. Tanck, B, Santos, A. Silveira, V. Santos, H. Santos, A. Santos, H. (1994). Evaluación de la población de oligoquetos (tierra) en las zonas degradadas. Revista de sector de ciencias agrarias. 13: 1-2, 35-40.

15. Domingo, J. (2010). El eucalipto y los suelos bajo clima mediterráneo. Departamento de ciencias Agroforestales Universidad de Huelva, España.

16. Fierro-Brunnenmeister, N., Gonzalez-Muñoz, N., Gallardo, A. \& Castro, P. (2009). Efectos de la descomposición de la hojarasca de especies nativas e invasoras en las propiedades del suelo. Quinto congreso forestal español, septiembre, 2009. Ávila, España.

17. Garcés, J., Peña, M., Cano-Santana, Z., Villeda, M. \& Orozco-Segovia, A. (2009). Cambios en la estructura de la vegetación derivados de acciones de restauración ecológica de las zonas de amortiguamiento Biológicas y Vivero Alto. (Tesis de pregrado). Universidad Nacional Autónoma de México - UNAM, México.

18. Huber, A., Iroume, A., Mohr, C. \& Frene, C. (2010). Efecto de plantaciones de Pinus radiata y Eucalyptus globulus sobre el recurso agua en la Cordillera de la Costa de la región del Biobío, Chile. Bosque, 31(3), 219-230.

19. Hussain, M., Gonzalez, L. \& Reigosa, M. (2007). Potencial alelopático de la especie invasora Acacia melanoxylon $\mathrm{R}$. Br en Galicia: Efecto sobre los índices de germinación. Universidad de Vigo, España. 
20. Instituto Distrital de Cultura de y Turismo. (2004). Bogotá: Panorama Turístico de 12 localidades. Alcaldía de Mayor de Bogotá. 20.

21. Instituto Nacional de los Recursos Naturales Renovables y del Medio Ambiente - INDERENA. (1976). Acuerdo 0030 de 1976. Recuperado de: http://www.alcaldiabogota.gov.co/sisjur/normas/Norma1.jsp?i=22591

22. Lobos, D. (2013) Evaluación temprana de restauración ecológica de bosques siempre verde en la Reserva Costera Valdiviana, Región de los Ríos. (Tesis de pregrado). Universidad Austral de Chile, Chile.

23. Manrique, O. (2009). Guía técnica para la restauración ecológica en áreas con plantaciones forestales exóticas en el Distrito Capital. Departamento Técnico Administrativo del Medio Ambiente - DAMA. Bogotá.

24. Ministerio de Ambiente y Desarrollo Sostenible. (2012). Plan Nacional de Restauración. Recuperado de: http:// www.andi.com.co/Ambiental/Documents/Plan_nacional_de_restauracion_Final_PDF.pdf

25. Ministerio de Ambiente, Vivienda y Desarrollo Territorial. (2005). Resolución 0463 de 2005. Recuperado de: http://www.alcaldiabogota.gov.co/sisjur/normas/Norma1.jsp?i=16272

26. Núñez, J. (2013). Respuestas eco fisiológicas y demográficas de Quercus ilex L. a alteraciones del balance facilitación / competencia del matorral en un ambiente semiárido. (Tesis doctoral). Universidad de Extremadura, España.

27. Osorio, J. (2009). Agua, montañas y ciudad. Los cerros orientales y Bogotá, abasto de agua y evolución ambiental. Bogotá; Instituto de estudios urbanos.

28. Oyarzun, C., Frene, C., Lacrampe, G., Huber, A. \& Herve, P. (2011). Propiedades hidrológicas del suelo y exportación de sedimentos en dos microcuencas de la Cordillera de la Costa en el sur de Chile con diferente cobertura vegetal. Bosque. 32 (1), 10-19.

29. Personería de Bogotá. (2007). Cerros orientales, desafío institucional. Recuperado de: https://mesacerros. files.wordpress.com/2007/10/cerros_orientales_desafio_institucional.pdf

30. Pinilla, A. Suarez, A. (1998). Efectos de plantaciones forestales sobre fauna y flora. CONIF, Bogotá, Colombia.

31. Quiroz, I., Pincheira, M., Hernández, J., Gonzalez, M., García, E. \& Soto, H. (2014). Efecto del volumen radicular sobre el crecimiento de Acacia en vivero y en terreno en el secano de la Región del Biobío, Chile. Revista Árvore, 38(1), 155-164.

32. Ríos, H. (2010). Coordinar el proyecto de restauración ecológica, rehabilitación y/o recuperación de áreas degradadas en el D.C. y la región que adelanta el Jardín Botánico de Bogotá. Recuperado de: http://jbb-repositorio. metabiblioteca.org:8080/bitstream/001/460/1/informe\%20 final\%20dic_ene11\%20HFRiosA\%20498_10.pdf

33. Rubiano, S. \& Garzón, R. (1989). Efectos del Eucalyptus globulus sobre la estabilidad estructural y otras propiedades físicas y biológicas de suelos de ladera en climas húmedo y seco. (tesis de pregrado) Universidad de Bogota Jorge Tadeo Lozano, Facultad de Agrologia. Bogota

34. Salamanca, B. \& Camargo, G. (2000). Protocolo Distrital de restauración ecológica: guía para la restauración de ecosistemas nativos en las áreas rurales de Santa Fe de Bogotá. Bogotá. DAMA. 288.

35. Santiago, M. \& Piedrahita, E. (2012). Efecto del Peso de la Semilla en el Crecimiento de Acacia melanoxylon $R$. $B r$ a los 6 Meses de Edad en Tres Condiciones de Suelo. Revista Facultad Nacional De Agronomía Medellín, 47(1), 125-143.

36. Solorza, J. (2011). Evaluación de la regeneración de Acacia decurrens, Acacia melanoxylon y Ullex europeus en áreas en proceso de restauración ecológica. Revista Luna Azul, (34), 66-80.

37. Souto, X., Gonzalez, L. \& Reigosa, M. (1993). Estudio de los efectos alelopáticos producidos por partes aéreas de distintas especies arbóreas (Eucalyptus globulus, Acacia melanoxylon, Quercus robur, Pinus radiata) en descomposición en el suelo. Conferencia llevada a cabo en el Congreso Forestal Español, Lourizan.

38. Vargas, O., Leon, O. \& Diaz, A. (2009). Restauracion ecológica en zonas invadidas por retamo espinos y plantaciones forestales de especies exóticas. (tesis de pregrado) Universidad Nacional de Colombia, Faculta de Ciencias Departamento de Biologia. 305 p.

39. Wiesner, D. (2007). Los caminos de los cerros. Bogotá: Multimpresos.

40. Wiesner, D. (2007). Plan director (Maestro) del corredor ecológico y recreativo de los cerros orientales Bogotá. Secretaria Distrital de Planeación. Recuperado de: http:// cerrosdebogota.org/elcorredor/assets/unidad-norte.pdf

\section{Conflicto de Intereses}

\author{
Los autores declaran no tener ningún conflicto \\ de intereses
}

